\title{
Off-diagonal disorder in the Anderson model of localization
}

\author{
P. Biswas ${ }^{1}$, P. Cain ${ }^{2}$, R. A. Römer ${ }^{2}$, and M. Schreiber ${ }^{2}$ \\ ${ }^{1}$ S N Bose National Center For Basic Sciences, Salt Lake City 700 091, Calcutta, India \\ Email: ppb@boson.bose.res.in; Tel.: +91333353057; Fax: +91333353477 \\ ${ }^{2}$ Institut für Physik, Technische Universität, D-09107 Chemnitz, Germany \\ Email: cain@physik.tu-chemnitz.de; Tel.: +493715313561; Fax: +493715313151
}

(October 24, 2018)

\begin{abstract}
We examine the localization properties of the Anderson Hamiltonian with additional off-diagonal disorder using the transfer-matrix method and finite-size scaling. We compute the localization lengths and study the metal-insulator transition (MIT) as a function of diagonal disorder, as well as its energy dependence. Furthermore we investigate the different influence of odd and even system sizes on the localization properties in quasi one-dimensional systems. Applying the finite-size scaling approach in conjunction with a nonlinear fitting procedure yields the critical parameters of the MIT. In three dimensions, we find that the resulting critical exponent of the localization length agrees with the exponent for the Anderson model with pure diagonal disorder.
\end{abstract}

Introduction. The electron localization properties of disordered solids are of high interest both experimentally and theoretically. A simple approach to this problem is given by the Anderson model of localization. In this model one considers a single electron on a lattice with $N$ sites described by the Hamiltonian

$$
H=\sum_{i \neq j}^{N} t_{i j}|i\rangle\left\langle j\left|+\sum_{i}^{N} \epsilon_{i}\right| i\right\rangle\langle i|
$$

where $|i\rangle$ denotes the Wannier states at site $i$. Disorder is usually introduced by varying the onsite potential energies $\epsilon_{i}$ randomly in the interval $[-W / 2, W / 2]$. In this work we report on the effects of additional off-diagonal disorder [1] [3], i.e., with random hopping elements $t_{i j} \in[c-w / 2, c+w / 2]$ between nearest neighbor sites. Thus $c$ represents the center and $w$ the width of the uniform off-diagonal disorder distribution. The energy scale is set by keeping $w=1$ fixed. This kind of disorder is similar to the random flux model [4,5], but here the modulus of the $t_{i j}$ is random while the phase is constant. The Hamiltonian (11) has a chiral symmetry on a bipartite lattice, i.e., under the operation $\psi_{n} \rightarrow(-1)^{n} \psi_{n}$, the Hamiltonian $H$ changes it sign and as a consequence, if $\epsilon_{n}$ is an eigenvalue of $H$, then so is $-\epsilon_{n}$.

We calculate the localization length $\lambda$ of the electronic wave function by the transfer-matrix method (TMM), which is based on an iterative reformulation [6] of Eq. (11) as

$$
\left(\begin{array}{l}
\psi_{n+1} \\
\psi_{n}
\end{array}\right)=T_{n}\left(\begin{array}{l}
\psi_{n} \\
\psi_{n-1}
\end{array}\right)=\left(\begin{array}{cc}
\mathbf{t}_{n+1}^{-1}\left(E \mathbf{1}-\mathbf{H}_{\perp}\right) & -\mathbf{t}_{n+1}^{-1} \mathbf{t}_{n} \\
\mathbf{1} & \mathbf{0}
\end{array}\right)\left(\begin{array}{l}
\psi_{n} \\
\psi_{n-1}
\end{array}\right),
$$

where $E$ is the energy, $\mathbf{H}_{\perp}$ is the Hamiltonian in the $n$th slice of the TMM bar, $\mathbf{1}$ and $\mathbf{0}$ are unit and zero matrix, respectively [7, and the diagonal matrix $\mathbf{t}_{n}$ represents the hopping elements connecting the $n$th with the $(n-1)$ th slice. The cross section of the bar is $1, M$, and $M^{2}$ for spatial dimension $d=1,2$, and 3 , respectively, and we always assume periodic boundary conditions for the TMM. Starting with two initial states $\left(\psi_{1}, \psi_{0}\right)$ one iterates along the quasi $1 \mathrm{D}$ bar until the desired accuracy is obtained. The localization length $\lambda_{d}=1 / \gamma_{\min }$ is computed from the smallest 
Lyapunov exponent $\gamma_{\text {min }}$ of the eigenvalues $\exp \left( \pm \gamma_{i}\right)$ of $\lim _{n \rightarrow \infty}\left(\tau_{n}^{\dagger} \tau_{n}\right)^{1 / 2 n}$ with $\tau_{n}=T_{n} T_{n-1} \cdots T_{2} T_{1}$. Assuming the validity of one-parameter scaling close to the MIT, we expect the reduced localization lengths $\lambda_{d}(M) / M$ to scale onto a scaling curve $\lambda_{d}(M) / M=f_{d}(\xi / M)$ with scaling parameter $\xi$.

Localization lengths at the 1D Dyson singularity. In 1D it has been shown that the density of states (DOS) of the disordered model diverges at $E=0$ [8,9], if $W=0$ on a bipartite lattice [3]. This singularity is intimately related to a divergence of the localization length [3, 10]. Assuming that the localization properties of the wave function can be described as usual by an exponential decay, one finds analytically 11]

$$
\lambda_{1}(E)=\frac{v_{F}}{D} \log \left|\frac{D}{E}\right|
$$

with $v_{F}$ the Fermi velocity and $D$ an energy parameter depending on the strength of the disorder. In Fig. 1 1 we show that this energy dependence is convincingly reproduced in numerical data of the model: $\lambda_{1}$ diverges at $E=0$ as in Eq. (3) for $W \rightarrow 0$. Note that the smallest values for the localization length are obtained for $c=0.25$. We remark that the validity of the exponential form of the wave function has been questioned in previous studies [3, 12] presenting evidence for power-law localization usually related to critical states.

Odd/even effects in quasi 1D systems. In 2D, the DOS also has a sharp peak at the band center [1] 3] and it was shown by the TMM that the localization length again diverges [13,14]. A finite-size-scaling (FSS) analysis of the TMM data together with studies of the participation numbers and multifractal properties of the eigenstates revealed [13,14 that the states at $E=0$ show critical behavior like in the 3D Anderson model at the MIT [15].

Renewed interest in the study of quasi 1D systems with off-diagonal disorder stems from the fact that the functional form of the divergence depends on whether $M$ is odd or even as shown by a Fokker-Planck approach in Ref. [10]. This question is intimately linked to the presence or absence of bipartiteness for the given lattice and boundary conditions. In Ref. [10] numerical data in support of the analytical results had already been shown. Here we will broaden the investigation by studying how the localization length at $E=0$ is influenced by the odd/even effects. Eilmes et al. 13,14 observed that the reduced localization length $\lambda_{2} / M$ for the 2D Anderson model with random hopping is independent of the system sizes at $E=0$ for bipartite lattices up to $M=200$. Motivated by the results of Refs. [3, 10], we show the behavior of the localization lengths for non-bipartite systems with odd $M$ as well as bipartite systems with even $M$ in Figs. 2 and 3 . We see that the values of the reduced localization length differ for odd and even $M$. Nevertheless, $\lambda_{2} / M$ at $E=0$ remains constant and thus critical in the sense of Ref. 13] for odd and even $M$ up to $M=101$ and 180, respectively.

Critical exponents in 3D. For the 3D model we computed the critical parameters at the MIT when either $E$ or $W$ are varied across the transition, i.e., $\xi \propto\left|E-E_{c}\right|^{-\nu_{E}}$ or $\xi \propto\left|W-W_{c}\right|^{-\nu_{W}}$ [16] at fixed $c$. The left panel of Fig. 囵 shows the reduced localization length $\lambda_{3} / M$ for even $M$ up to 14 at $E=0$ and $c=0$. The transition from extended states for $W \lesssim 4.05$ to localized states for $W \gtrsim 4.05$ is clearly visible as $\lambda_{3} / M$ decreases (increases) with increasing $M$ for the extended (localized) case. In the right graph the resulting scaling function $f_{3}(\xi / M)$ is plotted. It was obtained by a non-linear fit taking non-linear and non-universal corrections to FSS [17] into account. Comparing the exponents $\nu_{E}=1.61 \pm 0.07$ and $\nu_{W}=1.54 \pm 0.03$ of the transitions obtained from the fits at $W=0, c=0$ and $E=0$, $c=0$, respectively, with recent results for the Anderson model with pure diagonal disorder we find good agreement. Conclusion. In this work we have studied the localization properties of the Anderson model of localization with off-diagonal disorder. We find non-localized states in 1D and 2D only at the band center. In quasi 1D we examined 
odd/even system size effects for the localization length. We showed that the wave functions at $E=0$ in $2 \mathrm{D}$ remain critical up to the system sizes considered regardless of the odd/even effects. Our numerical results match reasonably well with the predictions [3, 10]. In the 3D case, we obtained the critical exponents of the MIT using TMM and FSS. Their values agree with recent results for the model with only diagonal disorder [17, 18]. Thus although the off-diagonal case exhibits some unusual features, its physics is nevertheless accurately described by the orthogonal universality class within the scaling theory of localization [6.19].

Acknowledgements. We thank C. M. Soukoulis and J. Stolze for pointing out Refs. [1].류 to us. 
[1] C. M. Soukoulis, I. Webman, G. S. Grest, and E. N. Economou, Phys. Rev. B 26, 1838 (1982).

[2] T. A. L. Ziman, Phys. Rev. B 26, 7066 (1982).

[3] M. Inui, S. A. Trugman, and E. Abrahams, Phys. Rev. B 49, 3190 (1994).

[4] M. Batsch, Ph.D. thesis, Universität Hamburg, 1997.

[5] M. Batsch, L. Schweitzer, I. K. Zharekeshev, and B. Kramer, Phys. Rev. Lett. 77, 1552 (1996), cond-mat/9607070.

[6] B. Kramer and A. MacKinnon, Rep. Prog. Phys. 56, 1469 (1993).

[7] A. MacKinnon and B. Kramer, Z. Phys. B 53, 1 (1983).

[8] F. J. Dyson, Phys. Rev. 92, 1331 (1953).

[9] T. P. Eggarter and R. Riedinger, Phys. Rev. B 18, 569 (1978).

[10] P. W. Brouwer, C. Mudry, and A. Furusaki, cond-mat/9904201 and cond-mat/9904200.

[11] R. H. McKenzie, Phys. Rev. Lett. 77, 4804 (1996).

[12] G. G. Kozlov, V. A. Malyshev, F. Domínguez-Adame, and A. Rodríguez, cond-mat/9805316.

[13] A. Eilmes, R. A. Römer, and M. Schreiber, Eur. Phys. J. B 1, 29 (1998).

[14] A. Eilmes, R. A. Römer, and M. Schreiber, phys. stat. sol. (b) 205, 229 (1998).

[15] H. Grussbach and M. Schreiber, Phys. Rev. B 57, 663 (1995).

[16] P. Cain, R. A. Römer, and M. Schreiber, Ann. Phys. (Leipzig) 8, SI-33 (1999), cond-mat/9908255.

[17] K. Slevin and T. Ohtsuki, Phys. Rev. Lett. 82, 382 (1999), cond-mat/9812065.

[18] A. MacKinnon, J. Phys.: Condens. Matter 6, 2511 (1994).

[19] E. Abrahams, P. W. Anderson, D. C. Licciardello, and T. V. Ramakrishnan, Phys. Rev. Lett. 42, 673 (1979). 


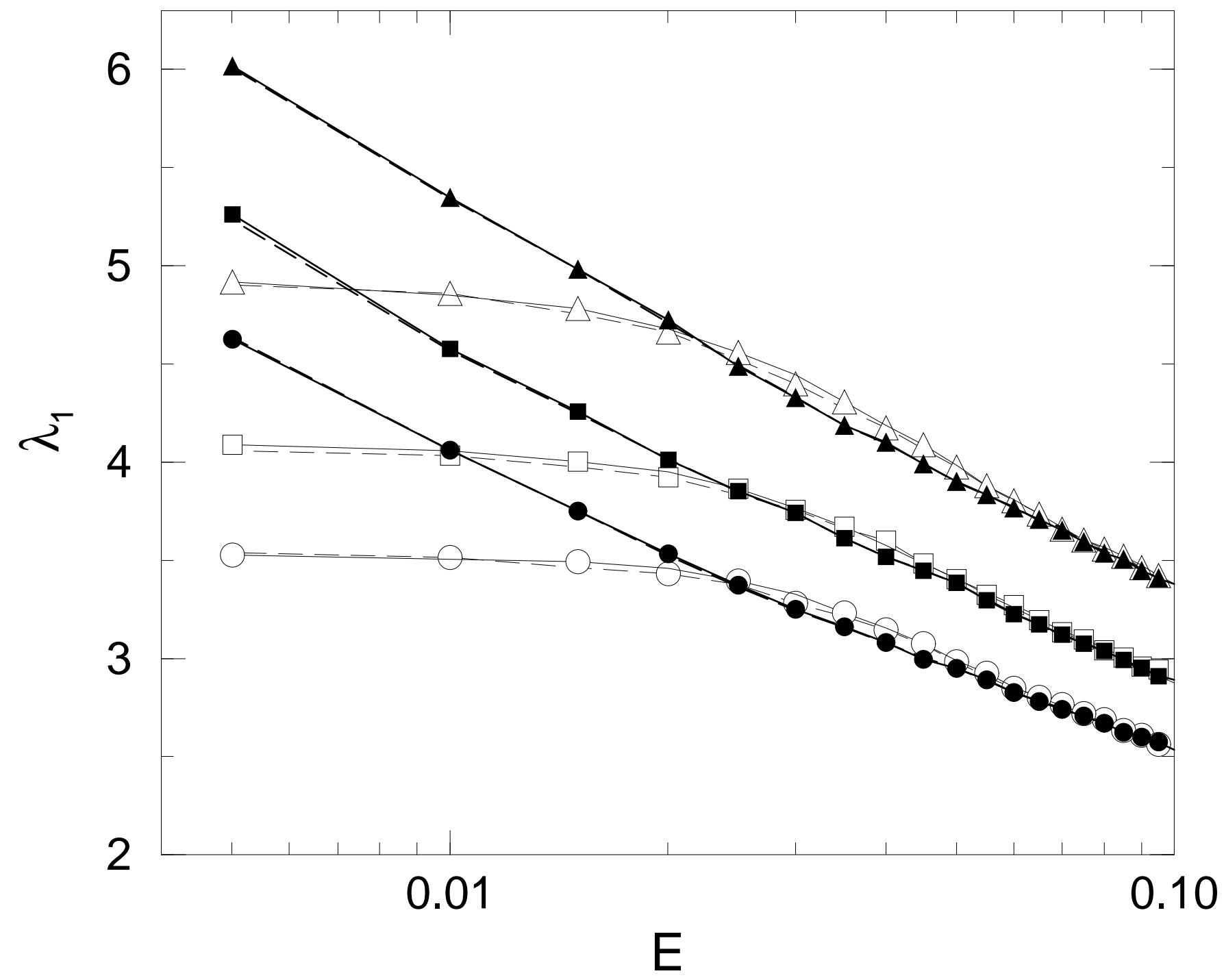

FIG. 1. Localization length $\lambda_{1}$ as function of energy $E$ with off-diagonal disorders $c=0(\square, \mathbf{\square}), 0.25(\circ, \bullet)$, and $0.5(\triangle, \mathbf{\Delta})$ for diagonal disorder $W=0.01$ (filled symbols) and 0.1 (open symbols) obtained by TMM with $1 \%$ accuracy. Solid (dashed) lines indicate data for $E>0(E<0)$. 


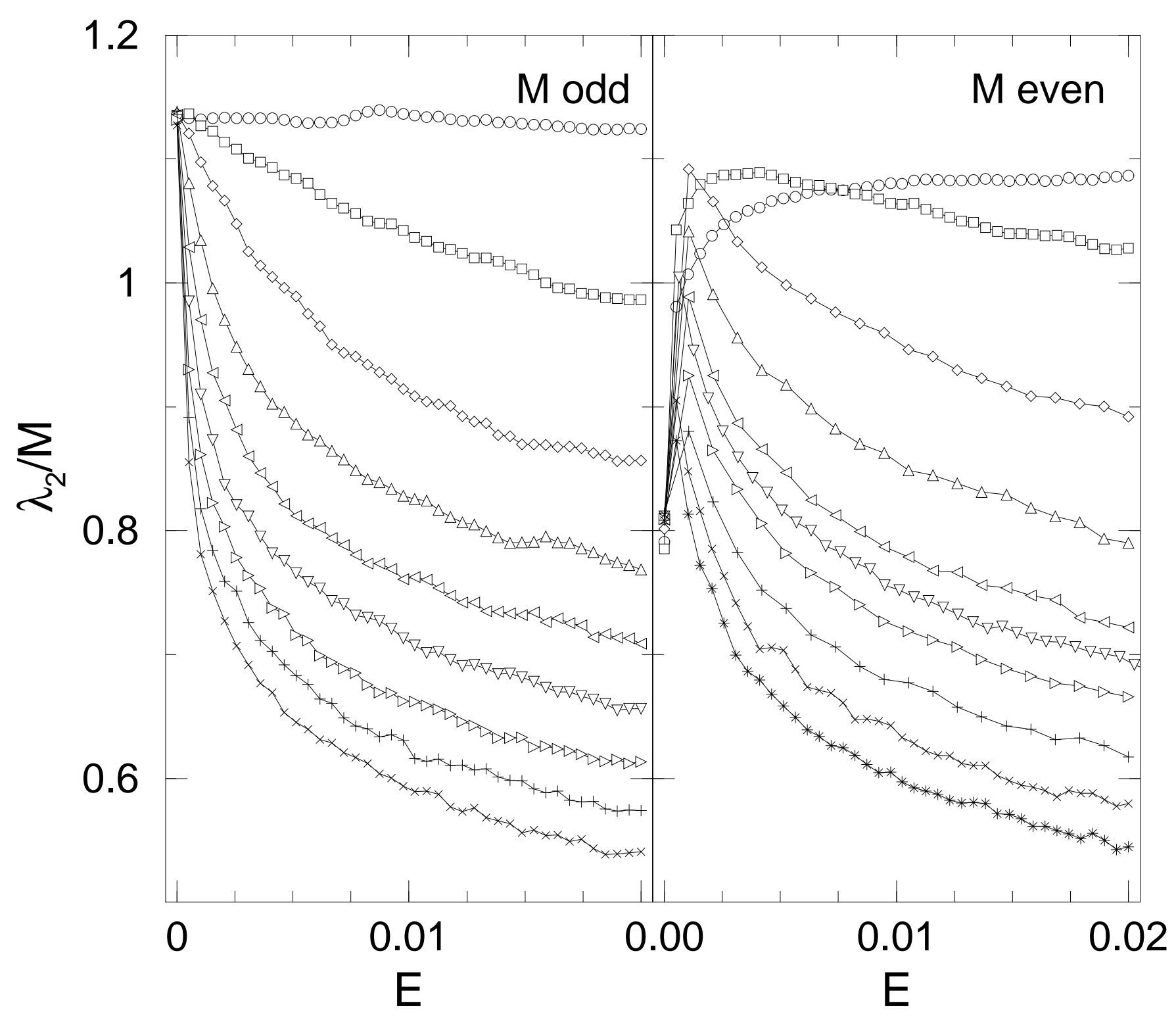

FIG. 2. Reduced localization lengths $\lambda_{2} / M$ as a function of energy for odd system sizes (left panel, $M=3,7, \ldots, 35$ from top to bottom) and even system sizes (right panel, $M=4,6,10,14,18,20,22,26,30,34$ from top to bottom) with random hopping for $c=0.25$. Note the strong decrease in $\lambda_{2} / M$ at $E=0$ for the bipartite even case. 


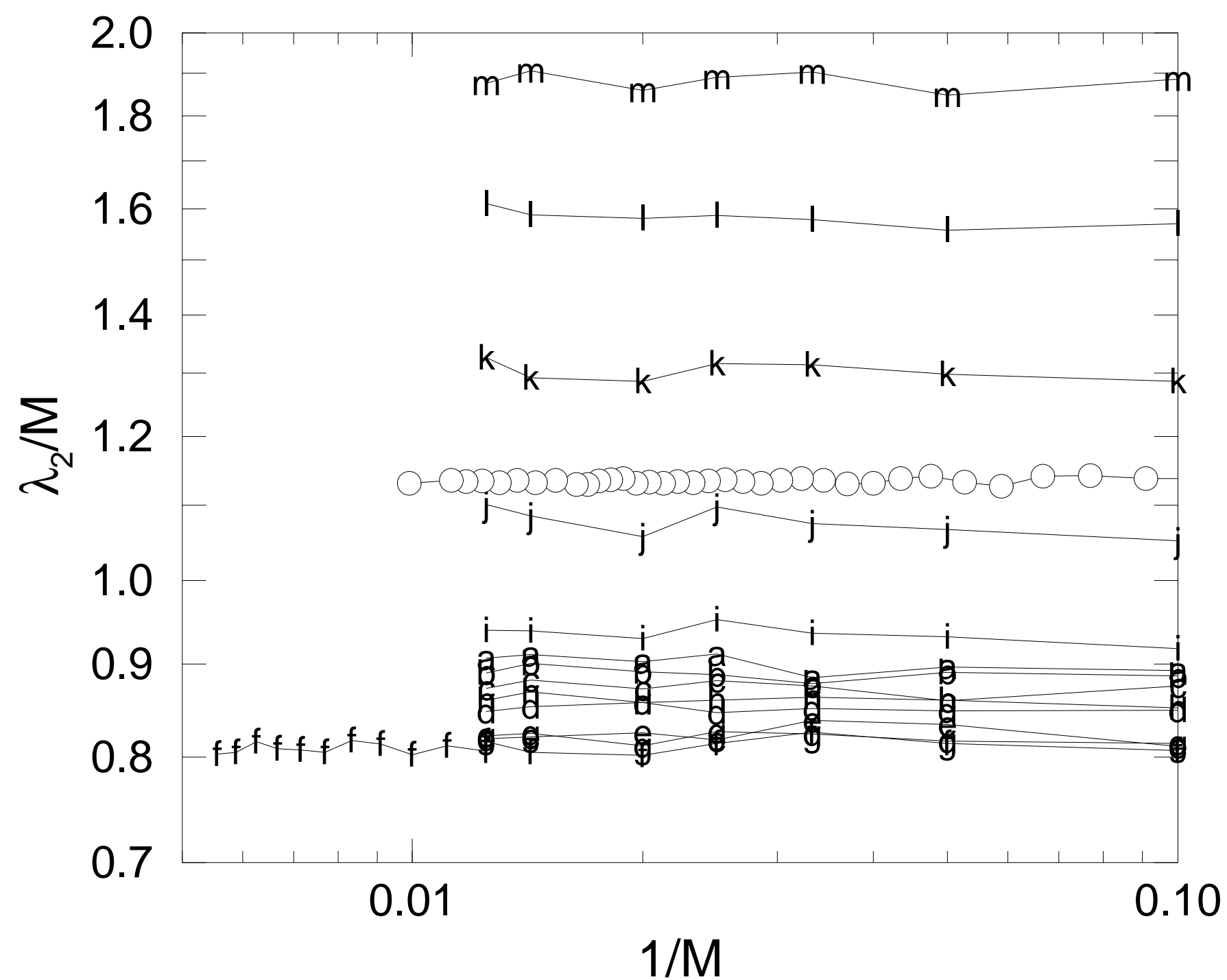

FIG. 3. Reduced localization length $\lambda_{2} / M$ vs. $1 / M$ for $E=0$ and even $M$ up to 180 with $1 \%$ accuracy corresponding to $c=0,0.05, \ldots, 0.25, \ldots 0.6$ as indicated by $\mathrm{a}, \mathrm{b}, \ldots, \mathrm{f}, \ldots, \mathrm{m}$ [13]. The circles represent data for odd system sizes $M=11,13, \ldots, 89$ and $M=101$ with $c=0.25$, i.e., the smallest $\lambda_{2} / M$ values, and $0.5 \%$ accuracy. 


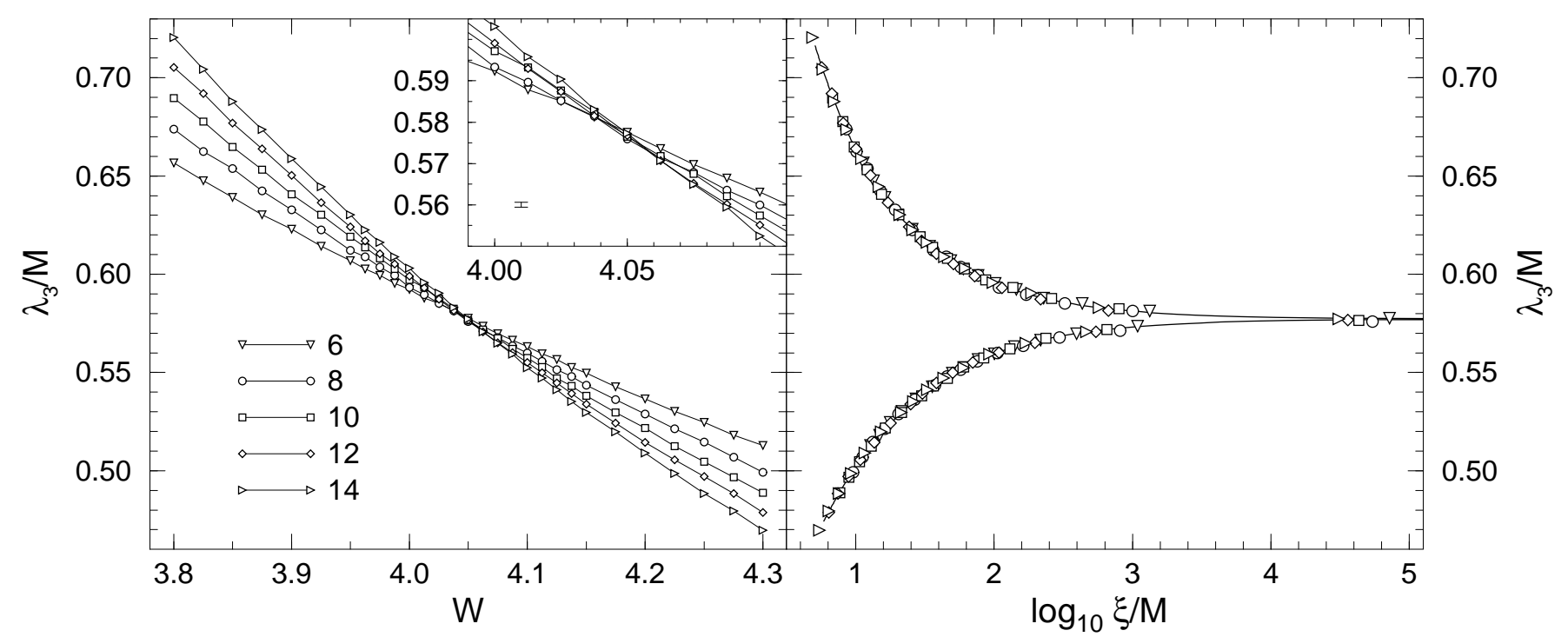

FIG. 4. Left: Reduced localization length $\lambda_{3} / M$ as function of diagonal disorder $W$ at $c=0$ and $E=0$ obtained by TMM with $0.1 \%$ accuracy. Note the MIT at $W_{c} \approx 4.05$. Inset: Enlarged region close to the MIT, the error bar is shown separately. Right: FSS curve for the TMM data from the left panel. 


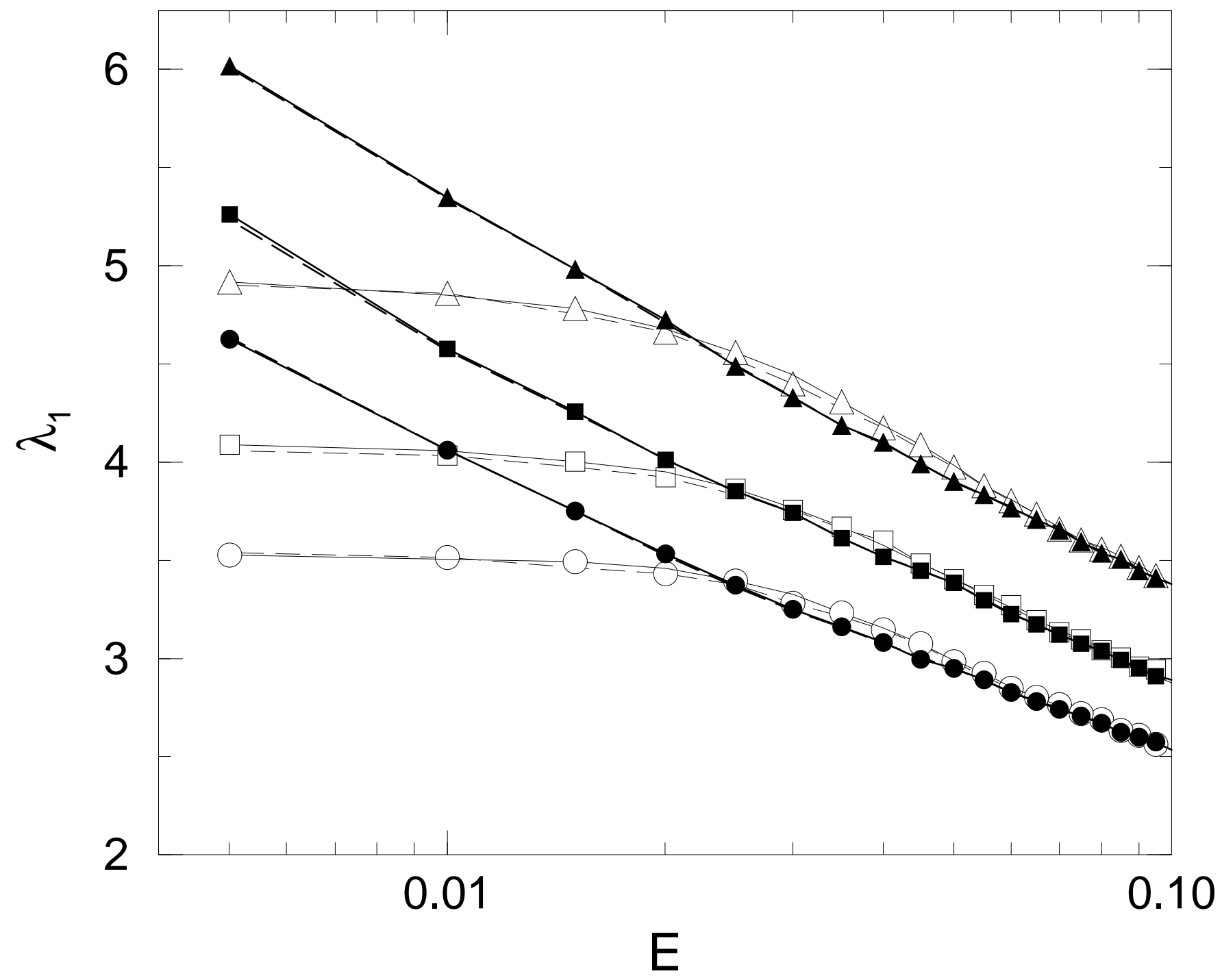




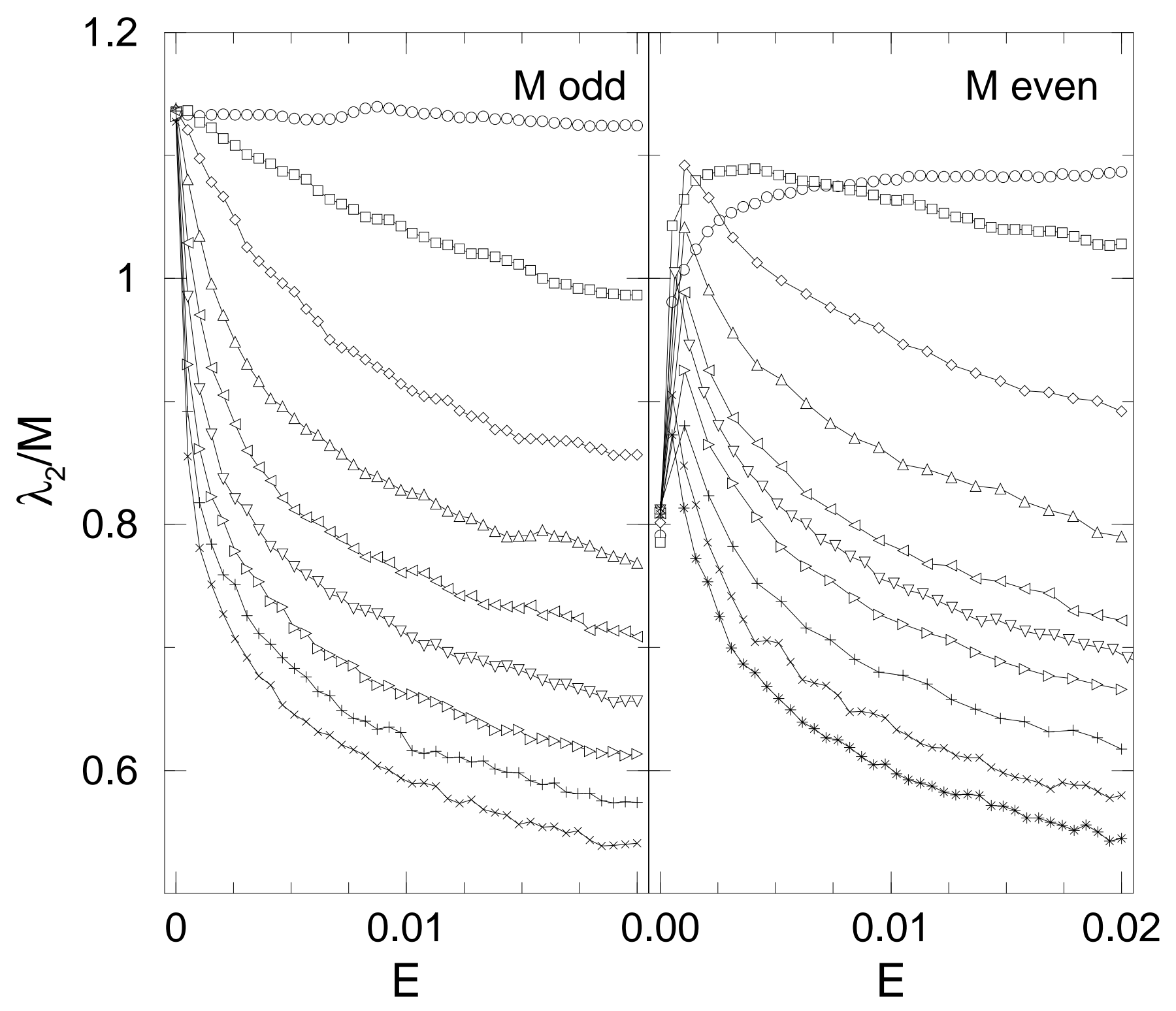




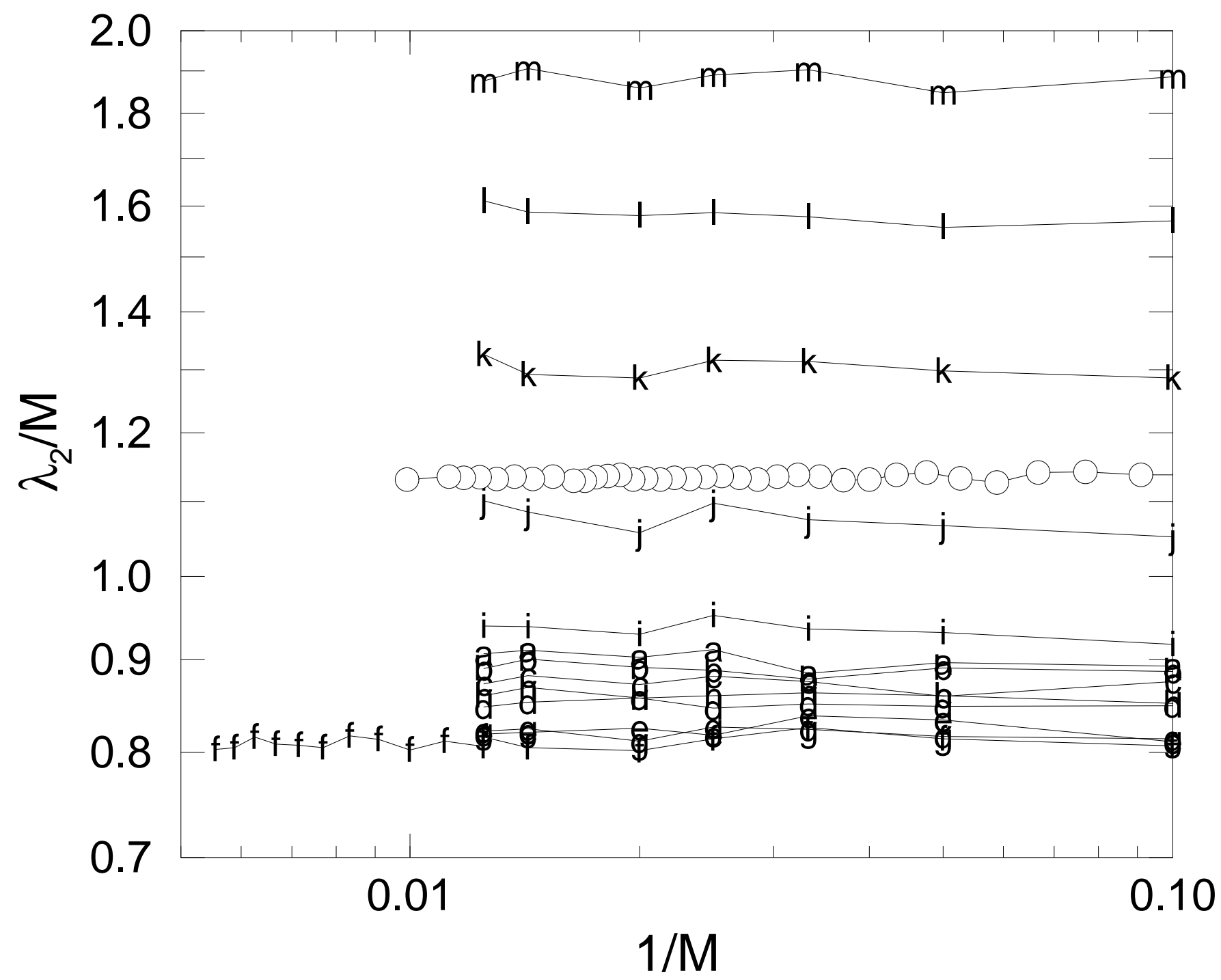




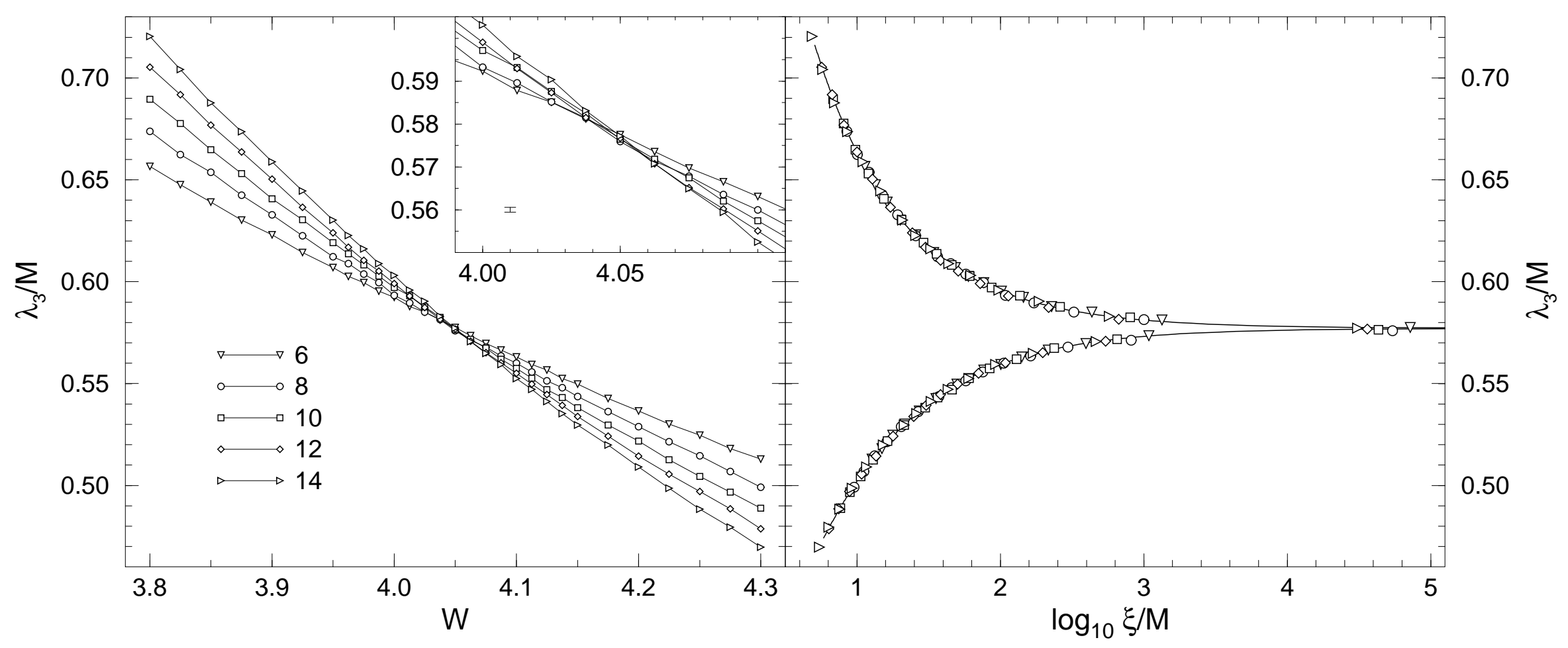

